\title{
Effet de l'intensité d'ensoleillement sur la croissance initiale en pépinière de Guibourtia ehie (A. Chev.) Leonard (Caesalpiniaceae), espèce couramment exploitée et menacée d'extinction, dans l'Unité de Gestion Forestière de Bossematié (Côte d'Ivoire)
}

\author{
Kouassi KOUADIO ${ }^{1 *}$, Hyppolite N'Da DIBI ${ }^{1}$, Lezin BOMISSO ${ }^{2}$ et \\ Rolande Bosson Kouassi ETTIEN ${ }^{1}$ \\ ${ }^{1}$ Laboratoire de Botanique et de Biologie Végétale, UFR Biosciences, Université Félix HOUPHOUET \\ BOIGNY Abidjan-Cocody; 22 bp 582 Abidjan 22, Côte d'Ivoire. \\ ${ }^{2}$ Laboratoire de Physiologie Végétale, UFR Biosciences, Université Félix HOUPHOUET BOIGNY Abidjan- \\ Cocody; 22 bp 582 Abidjan 22, Côte d'Ivoire. \\ *Auteur correspondant ; E-mail : attowoula@yahoo.fr; Tél : (+225) $47908275 / 04988960$.
}

\section{RESUME}

Une étude sur la croissance en pépinière de Guibourtia ehie (Caesalpiniaceae), en fonction de l'intensité de l'ensoleillement, a été réalisée dans l'Unité de Gestion Forestière de Bossematié. Le principal objectif est de contribuer à accroître les connaissances sur la croissance initiale de Guibourtia ehie (Caesalpiniaceae), espèce surexploitée et menacée d'extinction, afin de l'utiliser dans les programmes de reboisement des forêts ivoiriennes. L'étude est basée sur l'observation de la dynamique de croissance en hauteur et en diamètre et de la production foliaire de cette espèce dans des milieux d'ensoleillement d'intensité varié. Trois milieux d'intensité d'éclairement graduelle (faible, moyenne, forte) abritant chacun, des pépinières de Guibourtia ehie (Caesalpiniaceae) ont été installés et les différentes mensurations et dénombrements y ont été effectuées. Les résultats ont montré que les hauteurs moyennes des milieux faiblement et moyennement ensoleillés sont statistiquement différentes $(F=8,457 ; P=0,0070)$ de celle du milieu fortement ensoleillé. De même, les milieux peu ensoleillés ont eu une influence significativement positive $(F=9,636 ; P=0,0046)$ sur la croissance en diamètre des plants. Egalement, les milieux d'ensoleillement modéré ont eu une influence hautement significative $(F=12,64 ; P=0,0018)$ sur la production foliaire des jeunes tiges. Par ailleurs, l'étude a montré que la croissance initiale de Guibourtia ehie (Caesalpiniaceae) est lente, à l'instar de celle de la majorité des essences forestières couramment exploitées.

(C) 2013 International Formulae Group. All rights reserved.

Mots clés: Essence forestière, essence vulnérable, croissance en hauteur, croissance en diamètre, production foliaire.

\section{INTRODUCTION}

La régénération naturelle par graine désigne la capacité d'un peuplement végétal à se reconstituer spontanément, après enlèvement de tout ou une partie du couvert. Elle constitue le mode principal de la pérennisation d'une espèce. Cependant, les travaux de nombreux chercheurs tels que Ettien (2005) ; Kouadio et al. (2007) etc. ont montré la faible régénération naturelle par graines des espèces locales couramment exploitées dans les forêts classées ivoiriennes. 
Malheureusement, au cours des campagnes de reboisement de ces forêts, le choix est porté sur Tectona grandis Linn. (Verbenaceae), espèce exotique, au détriment des espèces locales pourtant, surexploitées. La conséquence directe de ce comportement est l'extinction des essences locales à diamètres exploitables. C'est le cas de Guibourtia ehie (Caesalpiniaceae) comme l'ensemble des espèces surexploitées dont les tiges sont devenues rares dans les forêts. Cette conséquence menace directement le secteur des industries du bois qui est pourtant pourvoyeur d'emplois. En effet, Kouadio (2012) a indiqué que sur les 43 espèces forestières locales couramment exploitées en Côte d'Ivoire, 25 sont inscrites sur la liste de l'UICN (2008). Cette liste concerne les espèces vulnérables, en dangers et à risque. Par ailleurs, peu d'études ont été réalisées sur la dynamique de croissance des paramètres biologiques (hauteur, diamètre et production de feuilles) de ces espèces. Aussi cette étude vient-elle pallier le déficit de connaissances sur les jeunes plants de Guibourtia ehie (Caesalpiniaceae).

L'objectif principal de l'étude est de contribuer à accroître les connaissances sur la croissance initiale en pépinière de Guibourtia ehie (Caesalpiniaceae), espèce locale surexploitée et menacée d'extinction, afin de les utiliser pour sa pérennisation, dans les forêts classées ivoiriennes. Il s'agira spécifiquement de déterminer la dynamique de croissance en hauteur, en diamètre et l'évolution de la phyllogénèse des jeunes tiges de cette espèce vulnérable, dans 03 milieux d'intensité lumineuse variée.

\section{MATERIEL ET METHODES Matériel}

L'étude a été réalisée dans le Département d'Abengourou, au Centre-Est de la Côte d'Ivoire, au sein de la pépinière de l'Unité de Gestion Forestière (UGF) de Bossematié-Appouessou. L'UGF est à $240 \mathrm{~km}$ d'Abidjan et est localisée entre $6^{\circ} 20^{\prime}$ et $6^{\circ}$ $35^{\prime}$ de latitude Nord et $3^{\circ} 20^{\prime}$ et $3^{\circ} 35^{\prime}$ de longitude Ouest (Figure 1).

Située dans le secteur mésophile du Domaine guinéen, le Département d'Abengourou est caractérisée par une température moyenne annuelle de l'ordre de 26,7 ${ }^{\circ} \mathrm{C}$ et par une pluviométrie moyenne annuelle de l'ordre de 1251,6 mm (Kouadio, 2007). Selon Kouadio et al. (2012), ce Département repose sur quatre types de sols : ferrasols, cambisols, arenosols et gleysols dont les plus dominants sont les ferrasols et la végétation est du type forêt semi-décidu.

\section{Méthodes}

La caractérisation de la dynamique de croissance de Guibourtia ehie (Caesalpiniaceae) en pépinière a nécessité la mise en place d'un dispositif comportant trois milieux d'ensoleillement d'intensité variée. Il s'agit d'un milieu fortement ensoleillé ou milieu ouvert, d'un milieu moyennement ensoleillé (ombrière constituée de claies légèrement recouverte de feuilles de palmier à huile) et d'un milieu faiblement ensoleillé (ombrière constituée de claies fortement recouverte de feuilles de palmier à huile). Chaque milieu est composé d'un bloc de 04 rangées de 10 sachets de semis chacune. L'expérience a été effectuée sur les plants de 04 semaines minimum, portant au moins 04 feuilles (Photographie 1) et a duré 90 jours. Chaque milieu comportait 40 échantillons saints. Sur chaque tige, le nombre de feuilles produites a été compté. Les mesures de hauteur ont été effectuées entre le collet et le bourgeon terminal de chaque plante, tandis que celles du diamètre ont été faites au niveau du collet. Les dénombrements et les mensurations ont été réalisés par semaines. Les rangées de sachets ont été arrosées 02 fois par jour au cours de l'expérience (le matin à 06 heures et le soir à 17 heures).

\section{Analyse des données}

Les variations des paramètres biologiques (croissance en hauteur, croissance en épaisseur et phyllogénèse) ont été traitées statistiquement par l'analyse de variance (ANOVA) à 5\% de seuil de significativité. Les tests de Tukey et de Newman Keuls ont été utilisés pour le classement des moyennes. Le logiciel Statistica 6.0 a permis de réaliser ces tests. Les vitesses de croissance en hauteur, en diamètre et le rythme d'émission foliaire ont été calculés à l'aide du logiciel Ecological Methodology. 

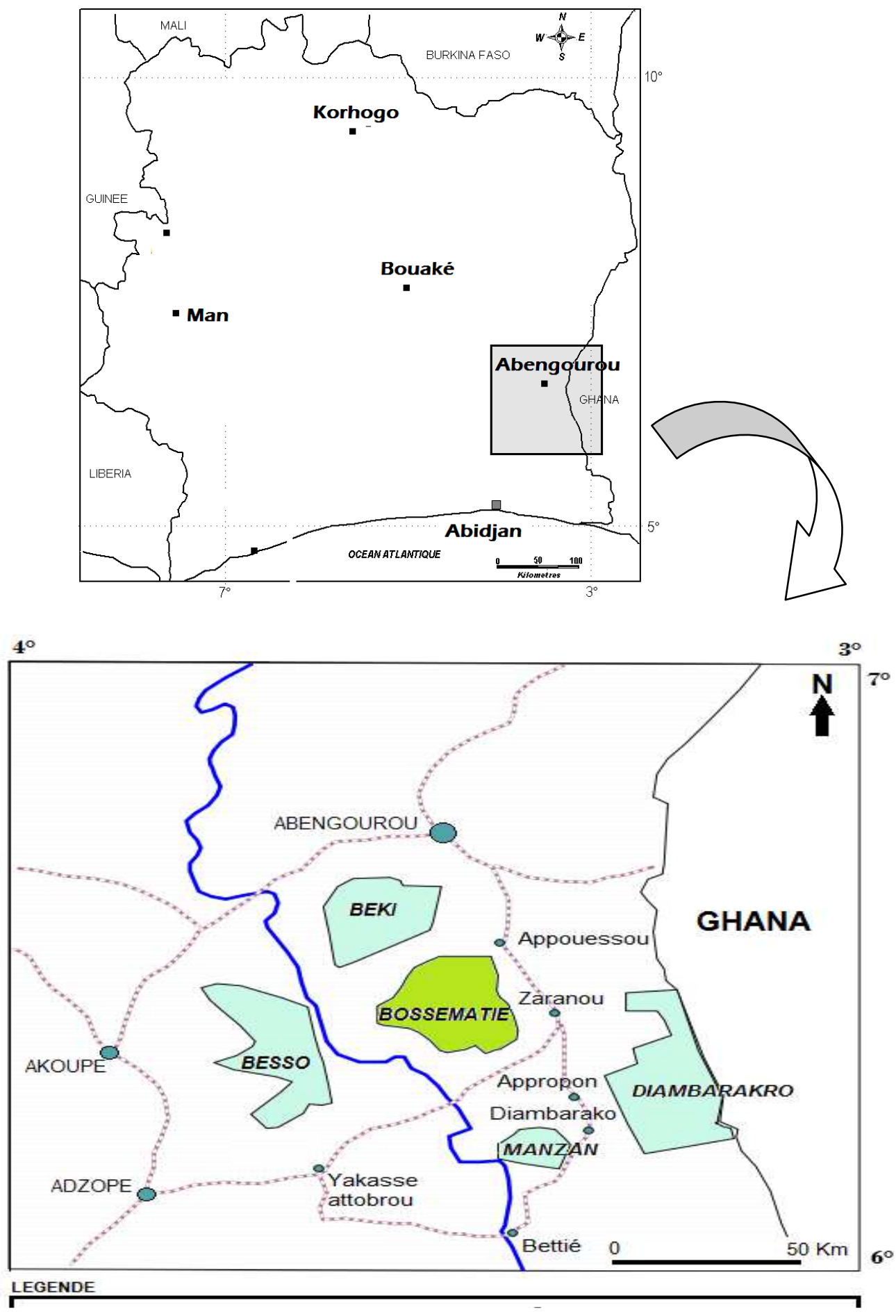

Figure 1 : Localisation de la forêt classée et de l'Unité de Gestion Forestière de Bossematié en Côte d'Ivoire. 


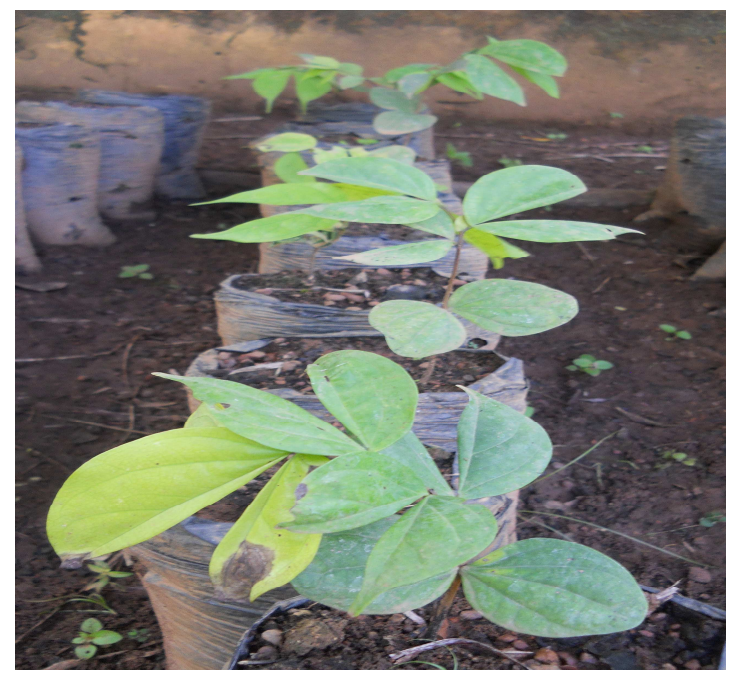

Photographie 1: Dispositif expérimental de l'essai de développement des jeunes tiges de Guibourtia ehie (Caesalpiniaceae) en condition de luminosité variée : Rangée de plants âgés de 08 semaines.

\section{RESULTATS}

Evolution de la hauteur des tiges en fonction de l'intensité de la lumière

L'élongation des jeunes tiges de Guibourtia ehie (Caesalpiniaceae) est accélérée dans les milieux d'éclairement modéré et est lente dans le milieu d'éclairement fort (Figure 2). En effet, la hauteur moyenne estimée dans ces milieux est de l'ordre de $17,2 \mathrm{~cm}$ et de $14,8 \mathrm{~cm}$, respectivement, dans les milieux moyennement et faiblement ensoleillés alors qu'elle est de l'ordre de 10,8 cm dans le milieu fortement ensoleillé, au terme des 12 semaines d'expérience. Par ailleurs, les croissances moyennes hebdomadaires estimées sont de l'ordre de 1,4 cm dans le milieu d'éclairement moyen, de 1,2 cm dans le milieu d'éclairement faible et de $0,8 \mathrm{~cm}$ dans le milieu fortement éclairé. Les résultats consignés dans le Tableau 1 montrent qu'il n'existe pas de différence significative entre le milieu faiblement éclairé et le milieu moyennement éclairé. Par contre, il existe une différence significative $(F=8,457 ; P=$ 0,0070) entre les résultats des milieux peu éclairés et ceux du milieu fortement ensoleillé.

\section{Evolution du diamètre des tiges en fonction de l'intensité de la lumière}

La croissance en diamètre des jeunes tiges de Guibourtia ehie (Caesalpiniaceae) est rapide dans les milieux faiblement et moyennement éclairés et est lente dans le milieu fortement éclairé (Figure 3). Les diamètres moyens des jeunes tiges sont de l'ordre de $0,22 \mathrm{~cm}$, de $0,21 \mathrm{~cm}$ et de $0,18 \mathrm{~cm}$, respectivement dans les milieux d'éclairement moyen, faible et fort. Par ailleurs, la croissance moyenne hebdomadaire est de l'ordre de $0,17 \mathrm{~cm}$ dans les milieux moyennement et faiblement ensoleillés tandis qu'elle est de l'ordre de $0,14 \mathrm{~cm}$ dans le milieu fortement éclairé. Les résultats du Tableau 2 montrent que les diamètres moyens des milieux faiblement et moyennement ensoleillés sont statistiquement différents $(F=$ 
9,636; $P=0,0046$ ) de celui du milieu fortement ensoleillé. Les diamètres moyens des milieux d'ensoleillement moyen et faible, ne présentent pas de différence significative.

\section{Evolution de la phyllogénèse des tiges en} fonction de l'intensité de la lumière

La production foliaire des jeunes tiges de Guibourtia ehie (Caesalpiniaceae) est abondante dans les milieux moyennement et faiblement ensoleillés tandis qu'elle est faible dans le milieu fortement ensoleillé (Figure 4). Trois mois après la levée, les jeunes tiges portent en moyenne 8,5 feuilles, 7,5 feuilles et 5,5 feuilles, respectivement, dans les milieux moyennement, faiblement et fortement éclairés. Le Tableau 3 montre une différence hautement significative $(F=12,64 ; P=$ 0,0018) entre les productions foliaires moyennes des milieux peu ensoleillés et la production foliaire moyenne du milieu fortement ensoleillé. Les productions foliaires moyennes des milieux d'éclairement moyen et faible ne présentent pas de différence significative.

Tableau 1 : Comparaison des hauteurs moyennes des jeunes plants de Guibourtia ehie (Caesalpiniaceae) des différents milieux d'éclairement.

\begin{tabular}{lc}
\hline Ensoleillement & Hauteurs moyennes \\
\hline Faible & $(146,99)$ \\
& $14,80 \pm 3,73^{\mathrm{b}}$ \\
\hline Moyen & $(173,52)$ \\
& $17,18 \pm 1,65^{\mathrm{b}}$ \\
\hline Fort & $(99,86)$ \\
& $10,82 \pm 2,33^{\mathrm{a}}$ \\
\hline F : test de décision de Fisher & P : probabilité $(\alpha=5 \%) ;$ Les moyennes accompagnées des mêmes lettres \\
ne sont pas significativement différentes au seuil de $\alpha=5 \%$.
\end{tabular}

Tableau 2 : Comparaison des diamètres moyens des jeunes plants de Guibourtia ehie (Caesalpiniaceae) dans les différents milieux d'éclairement d'intensité variée.

\begin{tabular}{lc}
\hline Ensoleillement & Diamètres moyens \\
\hline Faible & $(2,02)$ \\
& $0,21 \pm 0,01^{\mathrm{b}}$ \\
\hline Moyen & $(2,08)$ \\
& $0,22 \pm 0,01$ \\
\hline Fort & $(1,73)$ \\
& $0,18 \pm 0,01^{\mathrm{a}}$
\end{tabular}

F : test de décision de Fisher $\mathrm{P}$ : probabilité $(\alpha=5 \%)$; Les moyennes accompagnées des mêmes lettres ne sont pas significativement différentes au seuil de $\alpha=5 \%$. 
Tableau 3: Comparaison de la production foliaire moyenne des jeunes plants de Guibourtia ehie (Caesalpiniaceae) dans les différents milieux d'éclairement d'intensité variée.

\section{Ensoleillement}

\begin{tabular}{lc}
\hline Faible & $(69,33)$ \\
& $7,33 \pm 0,57^{\mathrm{b}}$ \\
\hline Moyen & $(79,2)$ \\
& $8,40 \pm 1,14^{\mathrm{b}}$ \\
\hline Fort & $(49)$ \\
& $5,40 \pm 0,89^{\mathrm{a}}$
\end{tabular}

$\mathrm{F}$ : test de décision de Fisher $\mathrm{P}$ : probabilité $(\boldsymbol{\alpha}=5 \%$; Les moyennes accompagnées des mêmes lettres ne sont pas significativement différentes au seuil de $\alpha=5 \%$.

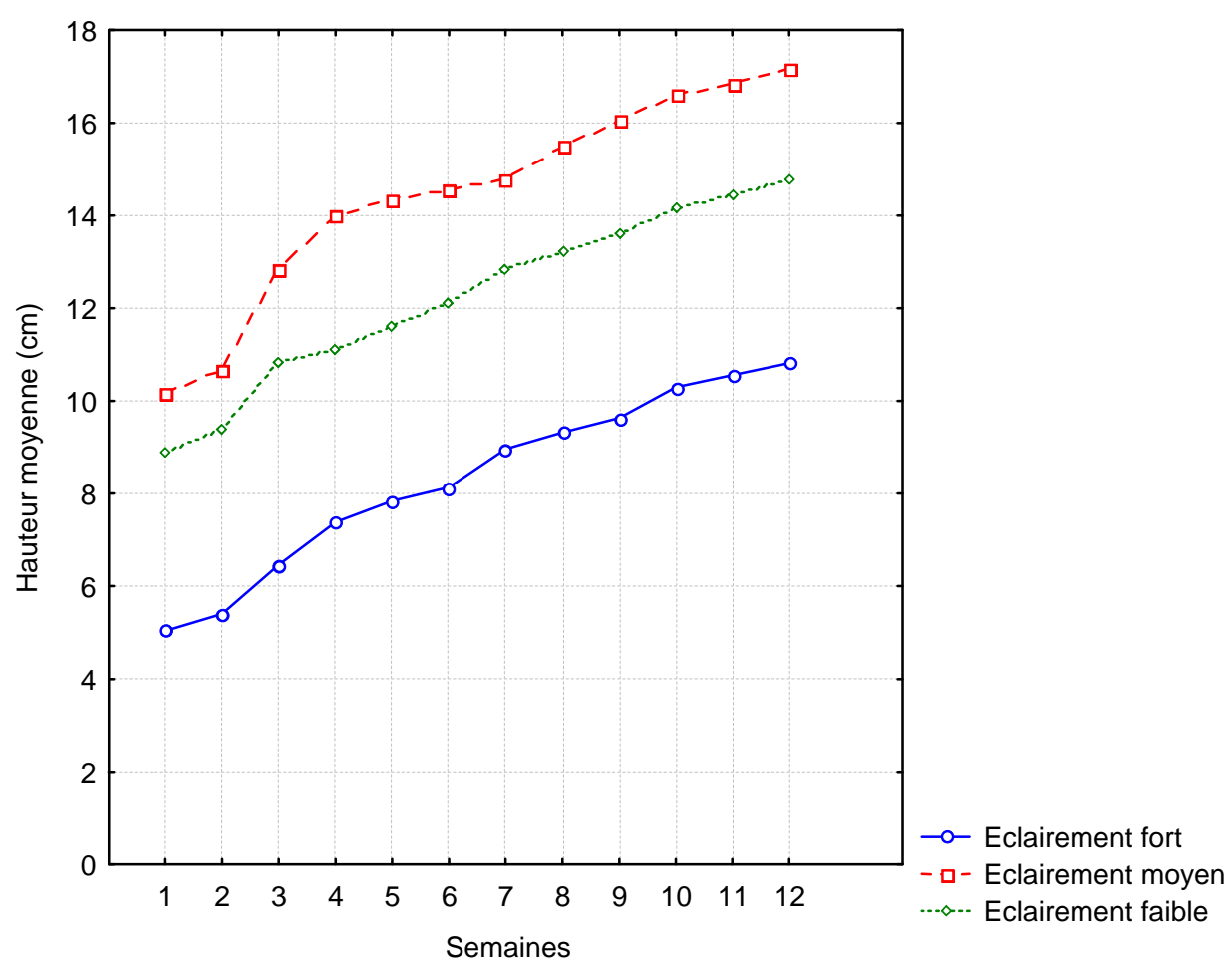

Figure 2: Courbes d'évolution de la hauteur moyenne des jeunes tiges de Guibourtia ehie (Caesalpiniaceae) en fonction du temps et de l'intensité d'ensoleillement. 


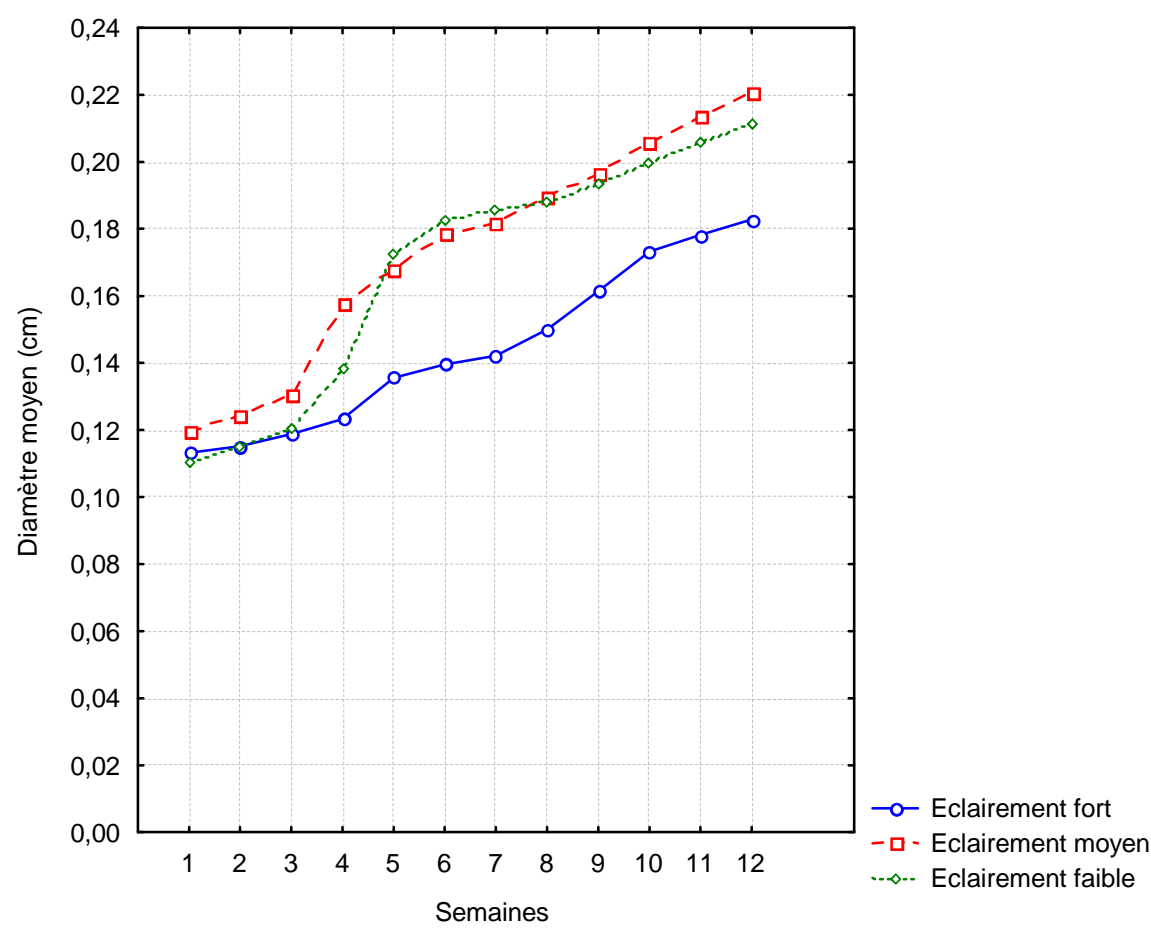

Figure 3: Courbes d'évolution du diamètre moyen des jeunes tiges de Guibourtia ehie (Caesalpiniaceae) en fonction du temps et de l'intensité d'ensoleillement.

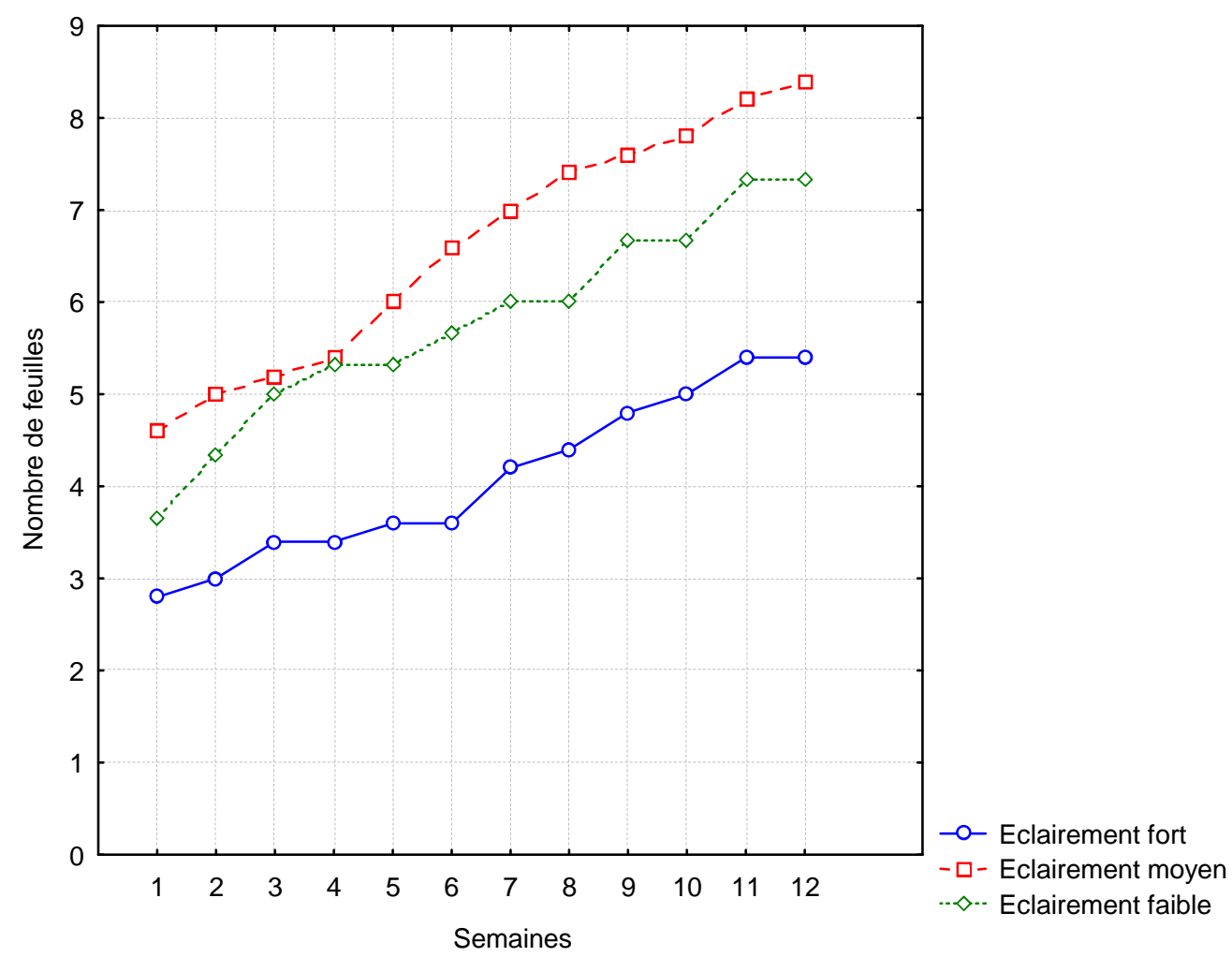

Figure 4 : Courbes d'évolution de la production foliaire moyenne des jeunes tiges de Guibourtia ehie (Caesalpiniaceae) en fonction du temps et de l'intensité d'ensoleillement. 


\section{DISCUSSION}

Trois mois après la levée, les jeunes tiges de Guibourtia ehie (Caesalpiniaceae) ont présenté une dynamique de croissance (hauteur, diamètre et production foliaire), meilleure dans les milieux moyennement et faiblement ensoleillés par rapport à celle observée dans le milieu fortement ensoleillé. Cette différence montre que la croissance de cette espèce est plus accélérée dans les milieux d'éclairement modéré. L'ensoleillement modéré stimule donc la croissance en hauteur, en diamètre et l'émission des feuilles des jeunes tiges chez Guibourtia ehie (Caesalpiniaceae). En effet, les vitesses de croissance en hauteur et en diamètre et le rythme d'émission foliaire des jeunes tiges ont été plus rapides dans les milieux d'éclairement modéré que dans le milieu d'éclairement fort, au cours des 90 jours d'expérience. Les éclairements modérés ont influencé donc positivement le développement des jeunes tiges de Guibourtia ehie (Caesalpiniaceae).

Comparé à d'autres espèces forestières aussi surexploitées et menacées d'extinction, la croissance initiale en hauteur et en diamètre de Guibourtia ehie (Caesalpiniaceae) est plus lente. En effet, les valeurs moyennes de la hauteur et du diamètre de la majorité de ces espèces sont plus élevées que celles de l'espèce étudiée. C'est le cas de Milicia excelsa (Welw.) C. C. Berg. (Moraceae) qui présente une hauteur moyenne de l'ordre de $25 \mathrm{~cm}$ et un diamètre moyen de l'ordre de $1,05 \mathrm{~mm}$, à 03 mois (database.prota.org/Milicia excelsa). Pour Entandrophragma cylindricum (Sprague) Sprague (Meliaceae), les valeurs moyennes sont de l'ordre de 19,26 cm pour la croissance en hauteur et de 3,6 $\mathrm{mm}$ pour la croissance en diamètre à 03 mois (database.prota.org/ Entandrophragma cylindricum). En définitive, la croissance initiale des essences forestières couramment exploitées est lente. Cette lenteur est probablement liée à leur tolérance vis-à-vis de la lumière. Quoique la germination de la plupart des semences d'arbres forestiers requière de la lumière, la croissance juvénile de la majorité de ces essences forestières qualifiée de «croissance lente», se déroule dans le sous-bois. Les fortes intensités lumineuses sont néfastes pour le développement des jeunes tiges. C'est le cas de Guibourtia ehie (Caesalpiniaceae) dont la croissance des jeunes tiges est accélérée dans les milieux d'éclairement modéré alors qu'elle est ralentie dans le milieu fortement éclairé. Par ailleurs, cette croissance initialement lente des espèces forestières pourrait être aussi liée, au développement du système racinaire comme l'on démontré Ouédraogo et al. (2006) sur Boswellia dalzelii Hutch. (Burseraceae). Dauro et al. (2003) avaient souligné déjà que le développement du système racinaire est un facteur déterminant dans la différence de survie des jeunes plants. L'hypertrophie de la racine reste une stratégie plus efficace pour la pérennisation des ligneux forestiers car elle permet de stocker des réserves nourricières. Malheureusement, d'après Guimbo Dan et al. (2011), le développement des racines est difficile et prend un temps considérable chez les essences forestières. Cette difficulté peut être un facteur limitant pour la nutrition des plants en eau et en sels minéraux, les rendant moins compétitif par rapport aux autres espèces du sous-bois. Tous ces facteurs rendent difficile la pérennisation des essences forestières. C'est pourquoi, selon Doucet et al. (2007), il est impératif d'améliorer la régénération des essences forestières par l'application des techniques sylvicoles. Il s'agit, dans les conditions naturelles, d'assister la régénération avec les techniques d'enrichissement (enrichissement en layon, enrichissement en placeaux et enrichissement des trouées d'abattage).

\section{Conclusion}

L'étude a montré que la croissance initiale en hauteur et en diamètre des jeunes tiges de Guibourtia ehie (Caesalpiniaceae) est lente, tout comme celle de la majorité des essences forestières couramment exploitées. Néanmoins, les croissances en hauteur, en diamètre et la phyllogénèse de cette espèce sont positivement influencées par les éclairements modérés. Le développement du système racinaire et la photosensibilité sont les principaux facteurs responsables de la croissance initiale lente de la majorité des 
essences forestières surexploitées. Pour faciliter la pérennisation des essences forestières en voie d'extinction, il est souhaitable d'introduire aussi Guibourtia ehie (Caesalpiniaceae) dans les programmes de reboisement des forêts classées ivoiriennes. Pour ce faire, les faibles intensités lumineuses sont plus appropriées à la production de plants de cette espèce. Dans la perspective d'une plus grande maîtrise de la croissance initiale des paramètres biologiques, l'étude doit s'étendre à l'ensemble des espèces forestières surexploitées et menacées d'extinction.

\section{REMERCIEMENTS}

Nous remercions les Responsables de la SODEFOR de nous avoir permis de réaliser nos travaux dans l'Unité de Gestion Forestière de Bossematié. Nous adressons aussi nos remerciements aux Responsables de l'Organisation Internationale des Bois Tropicaux (OIBT) de nous avoir accordé une bourse pour la réalisation de cette étude.

\section{REFERENCES}

Dauro D, Mohamed-Saleem MA, Gintzburger G. 2003. Recruitment and survival of native annual Trifolium species in the highlands of Ethiopia. Afr. J. Ecol., 34(1): 1-9.

Doucet JL, Dissaki A, Mengome A, Issembé Y, Dainou K, Gillet JL, Kouadio L, Laporte J. 2007. Dynamique des Peuplements Forestiers d'Afrique Centrale : Module de Formation. Faculté Universitaire des Sciences Agronomiques : Gembloux, Belgique; 133.

Ettien DT. 2005. Potentiels de régénération des essences forestières commerciales par la germination des graines, dans la forêt classée du Haut-Sassandra (Centre-Ouest de la Côte-d'Ivoire). Thèse de Doctorat $3^{\text {ème }}$ cycle, U.F.R. Biosciences, Université Abidjan-Cocody, Abidjan, Côte d'Ivoire, p. 259.
Guimbo Dan I, Ambouta MJK, Mahamane A, Larwanou M. 2011. Germination et croissance initiale de Neocarya macrophylla (Sabine) Prance, une espèce oléagineuse du Niger. Tropicultura, 29(2): 88-93.

Kouadio K. 2007. Études de la flore, de la végétation et de l'impact de l'éclaircie sélective par dévitalisation, sur les essences principales de la forêt classée de Bossematié, Est de la Côte-d'Ivoire. Thèse de Doctorat Unique, U.F.R. Biosciences, Université Abidjan-Cocody, Abidjan, Côte d'Ivoire, p. 192.

Kouadio K. 2012. Etude du comportement des essences locales exploitées couramment et menacées de disparition, en essaie de reboisement dans la pépinière de l'UGF de Bossematié, en fonction de l'intensité de l'ensoleillement. Rapport scientifique, Centre National de Floristique, UFR Biosciences, Université Félix HOUPHOUET-BOIGNY AbidjanCocody, Abidjan, Côte d'Ivoire, p. 57.

Kouadio K, Kouassi KE, Kouamé NF, Traoré D. 2007. Impact de l'éclaircie sur la régénération naturelle des essences principales, dans la forêt classée de Bossematié (Côte d'Ivoire). Sci. Nat., 4(1): 27-35.

Kouadio K, Kouassi KE, Dibi NH. 2012. Effet de l'éclaircie sélective sur la dynamique de la végétation arborescente dans la forêt classée de Bossematié (Côte d'Ivoire). Agron. Afr., 24(3): 219-230.

Ouédraogo A, Thiombiano A, Hahn-hadjali, Guiko S. 2006. Régénération sexuée de Boswellia dalzielii Hutch., un arbre médical de grande valeur au BurkinaFaso. B. Trop., 289(3): 41-52.

UICN. 2008. IUCN Red List of Threatened Species. www. iucnredlist.org. Visité le 15 Décembre 2008. 